\title{
Quantum real-space transfer in a heterostructure overgrown on the cleaved edge of a superlattice
}

\author{
Z. S. Gribnikov \\ Department of ECE, Wayne State University, Detroit, Michigan 48202, and Department of EECS, \\ University of Michigan, Ann Arbor, Michigan 48109
}

N. Z. Vagidov, ${ }^{\text {a) }}$ R. R. Bashirov, and V. V. Mitin

Department of ECE, Wayne State University, Detroit, Michigan 48202

G. I. Haddad

Department of EECS, University of Michigan, Ann Arbor, Michigan 48109

(Received 15 July 2002; accepted 27 September 2002)

\begin{abstract}
A dispersion relation for an electron in a two-layer (and also multilayer) quantum well (QW) is formed as a result of a certain combination of initial dispersion relations for each of the forming layers. Such a combination can be used to engineer new dispersion relations with desirable properties. The same relates to a two-dimensional electron gas (2DEG) induced in a multilayer medium. In this study, we consider first such a 2DEG in a specific two-layer structure where a superlattice (SL) plays the role of the second half-infinite layer, and electrons with large wave numbers along the SL vector spread from the first ordinary QW layer to this SL. As a result of such a quantum (dynamic) real-space transfer, electrons become heavier, and the dispersion relation achieves an additional negative effective mass (NEM) section. Such NEM dispersion relations were studied for several different material systems, including the two most interesting three-material systems: (1) an isomorphic $\mathrm{Al}_{0.15} \mathrm{Ga}_{0.85} \mathrm{As} / / \mathrm{GaAs} / \mathrm{Al}_{0.5} \mathrm{Ga}_{0.5} \mathrm{As}$ structure and (2) a strained $\mathrm{In}_{0.53} \mathrm{Ga}_{0.47} \mathrm{As} / / \mathrm{In}_{x} \mathrm{Ga}_{1-x} \mathrm{As} / \mathrm{In}_{y} \mathrm{Al}_{1-y}$ As structure $(x>0.53, y<0.52)$ with a strain-balanced $\mathrm{In}_{x} \mathrm{Ga}_{1-x} \mathrm{As} / \mathrm{In}_{y} \mathrm{Al}_{1-y} \mathrm{As}$ SL. Most of the results were verified using a simplified $1 \mathrm{D}$ model, but some of them were verified by more complicated 2D-model calculations. (C) 2003 American Institute of Physics. [DOI: 10.1063/1.1522814]
\end{abstract}

\section{INTRODUCTION}

Real-space transfer (RST) of electrons or holes can occur naturally in each layered heterostructure as a result of a parallel current transport in the layers. Such a transfer manifests itself in different forms depending on the thicknesses of the material layers forming the heterostructure, band discontinuities in heterojunctions, and other material and structural parameters. In accordance with this difference in manifestations, the RST requires different theoretical descriptions. This variety of forms and descriptions is limited from two opposite sides by two limiting cases, which can be named conditionally: (1) classic RST and (2) quantum RST. The classic RST, ${ }^{1,2}$ which has been researched and reviewed in great detail, ${ }^{3}$ assumes sufficiently large sizes of the layers forming the heterostructure. This assumption allows one to set definitely in each point of the structure all the dynamical parameters, including dispersion relations of electrons or (and) holes, and all the probabilities of scattering processes. The consequent step consists of a solution of kinetic equations in the structure with completely determined invariable dynamic parameters.

The opposite limiting case, the quantum RST, assumes such small sizes of the heterostructure layers that an electron cannot be described by local dispersion parameters. These

\footnotetext{
a)Electronic mail: nizami@nida.eng.wayne.edu
}

parameters must be determined by taking into account an electron motion simultaneously in several material layers. The simplest example of such a quantum RST is presented by an electron motion in the two-layer quantum well $(\mathrm{QW})$ with a potential profile shown in Fig. 1. This profile is characterized by the inner discontinuity $\delta\left(k_{T}\right)$ in the conduction band. Assuming that the ground quantization subband at $k_{T}$ $=0$ (where $\mathbf{k}_{T}=\left\{k_{Y}, k_{Z}\right\}$ is a wave vector in the plane of the $\mathrm{QW})$ is described by energy $\varepsilon_{I}$, the wave function of this state is localized primarily inside the right layer 1 (L1), and the dispersion relation $\varepsilon\left(k_{T}\right)$ must be described by effective mass $m_{1}$, which is relevant to L1. If effective mass $m_{2}$ for layer 2 (L2) is the same as $m_{1}$ and the confinement of the $\mathrm{QW}$ at the outer boundaries $x=w_{1}$ and $x=-w_{2}$ is large in comparison with $\delta(0)$, such a picture takes place for any $k_{T}$. But if mass $m_{2}$ differs from mass $m_{1}$ considerably, this picture can change substantially because $\delta\left(k_{T}\right) \neq \delta(0)$. For example, an increase in $k_{T}$ for $m_{2}>m_{1}$ leads to some decrease in $\delta\left(k_{T}\right)$. For a certain value $k_{T}=k_{T C}$, the discontinuity $\delta\left(k_{T}\right)$ can change sign: $\delta\left(k_{T C}\right)=0$. In this case, the wave function shifts from L1 to L2 more and more with an increase in $k_{T}$. This shift means that electrons become heavier and heavier because $m_{2}>m_{1}$. The described shift of the wave function from L1 to L2 with an increase in $k_{T}$ is the simplest example of the quantum RST (QRST). In contrast to a classic RST, which is a substantially dissipative (diffusive) effect (in fact, it is a form of thermionic emission), QRST is 


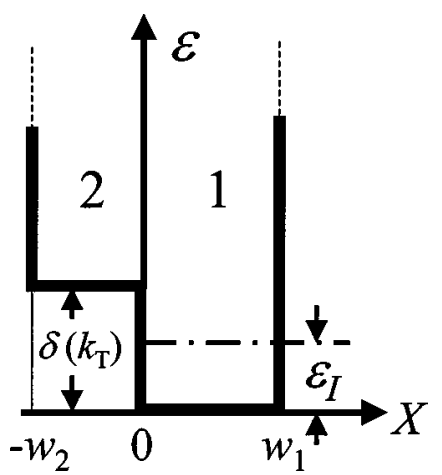

FIG. 1. Model of a two-layer heterostructure quantum well. The height of the inner heterobarrier $\delta$ (and as a result the ground quantization level position $\varepsilon_{I}$ ) depends on $k_{T}$.

a pure dynamic effect without any participation of scattering.

The result of QRST in the above-considered example is a combined electron dispersion relation, which includes electron parameters of both layers. This means that QRST can be a tool of quantum engineering to form artificial dispersion relations with desirable properties. However, for effective practical realization of such a kind of engineering, we need to combine initial dispersions with essentially different parameters (for example, $m_{2} \gg m_{1}$ ). To create a dispersion relation suitable for ballistic negative effective mass (NEM) oscillators, ${ }^{4,5}$ it is required that $m_{2} / m_{1}>2$. This is not a very simple requirement because (1) electrons in both L1 and L2 must be of the same $\Gamma$ valley, and (2) the heterostructure should be close to a perfect crystal.

Recently, ${ }^{6-8}$ it has been proposed to use a superlattice (SL) as a material for L2 in the heterostructure shown in Fig. 1. The vector of such a SL is directed along the QW plane (for example, along the $Z$ axis; see Fig. 2). The selection of a SL as a material for L2 allows one to control not only the effective mass $m_{2 Z Z}$ but also the effective discontinuity $\delta\left(k_{Y}, k_{Z}\right)$. Of course, the SL effective mass $m_{2 Z Z}$ can be made very large: much greater than mass $m_{1}$. Technologically, the heterostructure shown in Fig. 2 can be grown as a

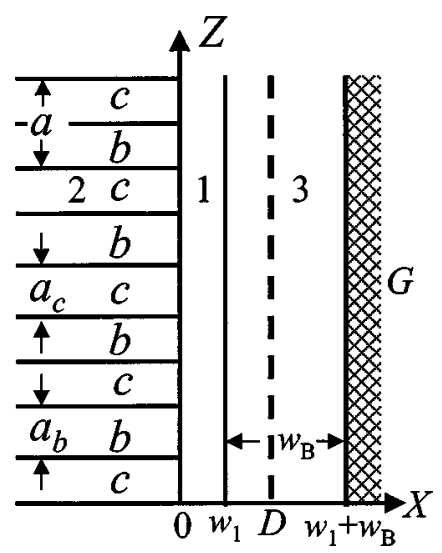

FIG. 2. Three-layer heterostructure with a 2DEG in the layers 1 and 2. The 2DEG is induced by the ionized donor sheet D in the barrier layer 3 (L3). The layer 1 (L1) is overgrown on the cleaved edge of the superlattice 2 (SL2) with SL barriers $b$ and SL QWs $c$. SL2 is doped by acceptors to restrict electron wave function spreading in SL2.

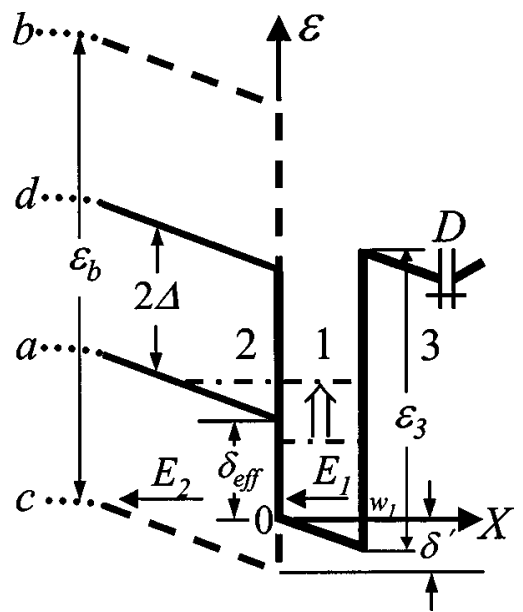

FIG. 3. Potential profile of the three-layer heterostructure depicted in Fig. 2. The position of the ground quantization level depends on $\left|k_{Z}\right|$. With an increase in $\left|k_{Z}\right|$ it shifts uphill relative to the miniband bottom as shown by the double arrow. The corresponding wave function spreads into SL2.

result of a secondary overgrowth on the cleaved edge of a $\mathrm{SL}^{9-11}$ [the so-called cleaved-edge-overgrowth (CEO) technology]. Since CEO technology will result in the infinite thickness of $w_{2}$ of L2 (the SL layer), a two-layer structure can be obtained on the basis of the two-dimensional electron gas (2DEG) induced in QW L1. This QW L1 is overgrown on the cleaved edge of the SL and followed by barrier layer 3 (L3). L3 contains a donor-doping sheet D and can be followed in turn by a gate G (Fig. 2).

The heterostructures, as shown in Fig. 2, have been fabricated by several groups (see Refs. 12-14 and references therein). Specifically, they are designed for Bloch oscillators and field-effect transistors. The potential profile appearing in such a structure is depicted in Fig. 3. It differs from a standard quasi-triangular 2DEG profile by the band discontinuity $\delta$ at $X=0$. This discontinuity is not the same as in Fig. 1 because curve $a$ in Fig. 3 indicates the bottom of the lowest miniband of SL quantization in SL2, which fills out all the half-space $X<0$. The curves $b, c$, and $d$, which are parallel to curve $a$, indicate, respectively, the bottom of a conduction band in the SL barriers, the bottom of a conduction band in the SL QWs, and the top of the same lowest SL miniband. The width of this miniband is equal to $2 \Delta$, and the total SL confinement is equal to $\varepsilon_{b}$. Since any real SL is a substantially anisotropic material, the transverse masses $m_{2 X X}$ $\cong m_{2 Y Y}$ can be much smaller than the longitudinal mass $m_{2 Z Z}$ (along the SL vector) and close to mass $m_{1}$. This means that a shifting of the electron wave function from L1 to SL2 and back is a fast process: an interface reflection does not hinder it.

In order to restrict the length of the SL2 region in which the wave function can spread from L1 to SL2, the latter is doped by acceptors. As a result of this doping, a quasihomogeneous electric field of a heterostructural $p n$ junction appears, and this field impedes the shift of the electron wave function into $p$-type SL2. An equilibrium distribution of this field is determined by both the value of the acceptor concentration in SL2 and a value of the modulation donor doping in the barrier layer 3. A potential of back $p^{+}$contact on the left 
side of the SL2 and a gate potential in the gate G can serve as additional regulators of the above-mentioned electric field, which confines the 2DEG from the left side.

This work is devoted to calculations of electron dispersion relations $\varepsilon=\varepsilon\left(k_{Y}, k_{Z}\right)$ for the 2DEG in the heterostructure with the potential profile depicted in Fig. 3. All the calculated dispersion relations contain the NEM sections, which differ from one another by location, form, and size. We have selected ones which are most suitable from the point of view of NEM ultrahigh frequency (UHF) oscillators. Methods of these calculations are considered and discussed in Sec. II. In Sec. III we present results of our calculations for several selected unstrained and strained heterostructures. In Secs. IV and V we present a short discussion of the results and concluding remarks.

\section{COMPUTATIONAL PROCEDURE}

A direct method to calculate electron dispersion relations for the described heterostructures consists of solutions of the Schrödinger equation in each of the semiconductor regions divided by abrupt heterostructural boundaries. These solutions must be joined (sewed together) by suitable boundary conditions. Such a procedure foresees a separate consideration of not only QW L1, covering barrier L3, but also each of the barriers $b$ and the QWs $c$ forming SL2 (see Fig. 2). This means that the computational procedure is substantially two-dimensional and requires a progressive decrease in the size of the spatial grid meshes for a decrease in thicknesses of the above-mentioned barriers $b$ and QWs $c$. We have implemented such a procedure in the one-conduction-band approximation (assuming that all the considered barrier discontinuities are small in comparison to the band-gap value $\left.\varepsilon_{g}\right)$. We have also assumed that no electric field exists along the SL vector: $E_{Z}=0$ (a flatband approximation). In L1 and in SL2, we assume homogeneous electric fields $E_{1}$ and $E_{2}$, respectively, which are directed along the $X$ axis. As mentioned above, the field $E_{2}$ restricts a spreading of the electron wave function deep into SL2. The procedure of calculations described here can be related to the generalized KronigPenney (KP) model. Along with the heterostructure shown in Figs. 2 and 3, we have completed the analogous calculations of electron dispersion relation $\varepsilon=\varepsilon\left(k_{Y}, k_{Z}\right)$ for the simpler subsidiary heterostructure that contains an SL layer 2 with a restricted size $w_{2}$ (see Fig. 4). This size $w_{2}$ has been varied in a wide range (as a rule $w_{2} \geqslant 3 w_{1}$ ). We have mainly used the calculations on the basis of the above-described generalized KP model (which is called the 2D model below) in order to numerically substantiate another much simpler model (which has been used for calculation of most of our results). In this simplified model (called the 1D model), the SL is considered separately, and it is presented by electron dispersion relations in its minibands:

$$
\varepsilon=\varepsilon_{n}^{(\mathrm{SL})}\left(k_{Z}, k_{Y}\right) .
$$

Although the results presented below use only the KP model calculations, the dispersion relations (1) can be obtained in arbitrary approximations including much more accurate ones. The relation (1) allows one to consider SLs as

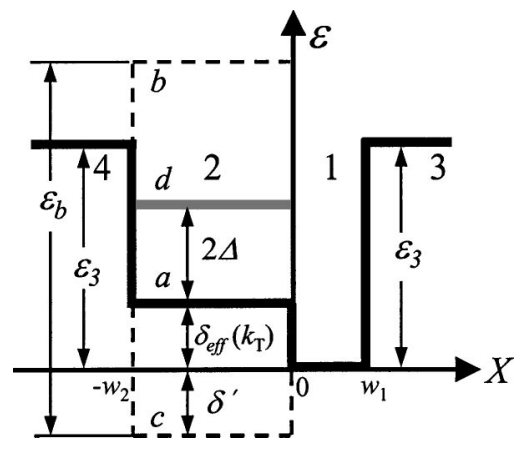

FIG. 4. Potential profile of the hypothetical subsidiary heterostructure (without electric fields $E_{1}$ and $E_{2}$ and with the additional hypothetical barrier layer 4).

separate building elements of a heterostructure just as we consider separate Bloch crystals, which together form a certain complicated heterostructure. Such an approach requires satisfying the strong inequality

$$
\delta r \gg a,
$$

where $a$ is a period of an SL, and $\delta r$ is a characteristic size of (or inside of) a heterostructure. It may be a size like $w_{1}$ and $w_{2}$ in Fig. 1, $w_{1}$ in Fig. 3, or a size of the ground quantization state for a $2 \mathrm{DEG}$ in a triangular potential. Considering an SL as a certain separate element of a heterostructure, we need to formulate boundary conditions for electron wave functions in abrupt boundaries between SLs and homogeneous semiconductor regions. Note that the number of such boundaries decreases substantially in comparison with the generalized KP model approach. For example, in the structure shown in Fig. 2, we have only three boundaries: $X=0, X=w_{1}$, and $X=w_{1}+w_{B}$. All the boundaries inside of the SL are taken into account in the relations (1). As a whole, the spatially inhomogeneous problem loses its twodimensionality and become 1D: inhomogeneity takes place only along the $X$ axis (1D model).

Below, we implement the above-indicated procedure only in the simplest case, when (1) we can take into account only the single (the lowest) miniband of the SL quantization (neglecting all the higher minibands), and (2) a dispersion relation in this miniband can be written in the additive form:

$$
\varepsilon=\varepsilon_{\mathrm{SL}}\left(k_{Z}, \mathbf{k}_{\perp}\right)=\varepsilon_{\mathrm{SL}}\left(k_{Z}, 0\right)+\hbar^{2} k_{\perp}^{2} / 2 m_{\perp},
$$

where $\mathbf{k}_{\perp}=\left\{k_{X}, k_{Y}\right\}, k_{\perp}^{2}=k_{X}^{2}+k_{Y}^{2}$. In many cases (but not always), we have

$$
\varepsilon_{\mathrm{SL}}\left(k_{Z}, 0\right)=\varepsilon_{\mathrm{SL}}(0,0)+\Delta\left(1-\cos k_{Z} a\right),
$$

where $a$ is the SL period, and $\Delta$ is a half of the miniband width. For the KP model, the relation (3) can be approximately obtained from the equation ${ }^{15}$

$$
\begin{aligned}
\cos k_{Z} a= & \cos \kappa_{c} a_{c} \cosh \lambda_{b} a_{b} \\
& +\frac{m_{c}^{2} \lambda_{b}^{2}-m_{b}^{2} \kappa_{c}^{2}}{2 m_{b} m_{c} \lambda_{b} \kappa_{c}} \sin \kappa_{c} a_{c} \sinh \lambda_{b} a_{b},
\end{aligned}
$$

where $\kappa_{c}^{2}=\left(2 m_{c} / \hbar^{2}\right) \varepsilon_{\mathrm{SL}}\left(k_{Z}, \mathbf{k}_{\perp}\right)-k_{\perp}^{2}, \quad \lambda_{b}^{2}=2 m_{b} / \hbar^{2}\left[\varepsilon_{b}\right.$ $\left.-\varepsilon_{\mathrm{SL}}\left(k_{Z}, \mathbf{k}_{\perp}\right)\right]-k_{\perp}^{2}, m_{c}$ and $m_{b}$ are the electron effective masses in the SL QWs and barriers, respectively, along the 
SL vector, and $\varepsilon_{b}$ is the height of the SL barriers (the SL confinement). To use Eq. (3), we need to calculate $m_{\perp}$ from Eq. (5) numerically. To use Eq. (4), we also need to determine numerically the parameter $\Delta$ from Eq. (5) (see some details in Ref. 16). But it must be emphasized again that the above-described procedure has no direct connection with either the KP model or Eq. (5). The values of the mass $m_{\perp}$ or parameters describing $\varepsilon_{\mathrm{SL}}\left(k_{Z}, 0\right)$ can be calculated from any other model (including very sophisticated ones) or obtained empirically.

Equation (3) allows us to use in the boundary $X=0$ the standard boundary conditions:

$$
\begin{aligned}
& \Psi_{1}(+0)=\Psi_{2}(-0), \\
& \left.\left(1 / m_{1}\right)\left(\partial \Psi_{1} / \partial X\right)\right|_{X=+0}=\left.\left(1 / m_{\perp}\right)\left(\partial \Psi_{2} / \partial X\right)\right|_{X=-0},
\end{aligned}
$$

where wave function $\Psi_{1}(X)$ relates to electrons in L1 with effective mass $m_{1}$ and wave function $\Psi_{2}(X)$ relates to electrons in SL2 with transverse effective mass $m_{\perp}$. The wave equations for the functions $\Psi_{1,2}(X, Y, Z)$ are

$$
\begin{aligned}
& -\frac{\hbar^{2}}{2 m_{1}}\left(\frac{\partial^{2}}{\partial Z^{2}}+\frac{\partial^{2}}{\partial \mathbf{r}_{\perp}^{2}}\right) \Psi_{1}-e E_{1} X \Psi_{1}=\varepsilon \Psi_{1}, \\
& \varepsilon_{\mathrm{SL}}\left(-i \hbar \frac{\partial}{\partial Z}, 0\right) \Psi_{2}-\frac{\hbar^{2}}{2 m_{\perp}} \frac{\partial^{2} \Psi_{2}}{\partial \mathbf{r}_{\perp}^{2}}-e E_{2} X \Psi_{2}=\varepsilon \Psi_{2},
\end{aligned}
$$

where $E_{1,2}$ are the above-mentioned electric fields directed along the $X$ axis (Fig. 3), $\mathbf{r}_{\perp}=\{X, Y\}$. The wave functions $\Psi_{1,2}(X, Y, Z)$ are selected in the form:

$$
\Psi_{1,2}(X, Y, Z)=\psi_{1,2}\left(X, k_{Y}, k_{Z}\right) \exp \left(i k_{Y} Y+i k_{Z} Z\right),
$$

where functions $\psi_{1,2}\left(X, k_{Y}, k_{Z}\right)$ are defined by the equations

$-d^{2} \psi_{1} / d X^{2}=\left[\left(2 m_{1} / \hbar^{2}\right)\left(\varepsilon+e E_{1} X\right)+k_{Y}^{2}+k_{Z}^{2}\right] \psi_{1}$,

$-d^{2} \psi_{2} / d X^{2}=\left\{\left(2 m_{\perp} / \hbar^{2}\right)\left[\varepsilon-\varepsilon_{\mathrm{SL}}\left(k_{Z}, 0\right)+e E_{2} X\right]+k_{Y}^{2}\right\} \psi_{2}$,

which must be solved with boundary conditions in the plane $X=0$ analogous to Eqs. (6a) and (6b). In the plane $X=w_{1}$, function $\psi_{1}$ is sewed to function $\psi_{3}$, which satisfies in layer 3 to the wave equation with isotropic effective mass $m_{3}$, and we assume for simplicity that $E_{3}=0$. This assumption does not lead to a noticeable error because the heterobarrier height $\varepsilon_{b}^{\prime}$ in this plane is large.

The same equations are also solved for the subsidiary heterostructure shown in Fig. 4. This hypothetical structure does not contain any electric fields $E_{1}=E_{2}=E_{3}=0$ but includes one more heterojunction at $X=-w_{2}$, where the heterobarrier is always comparatively high.

\section{SELECTED RESULTS FOR THE SELECTED HETEROSTRUCTURES}

\section{A. Description of the structures}

Selection of specific materials for L1 and SL2 was based on the following factors. First, to provide high power of the expected UHF oscillations and the desirable working temperatures, we would like to obtain the calculated dispersion

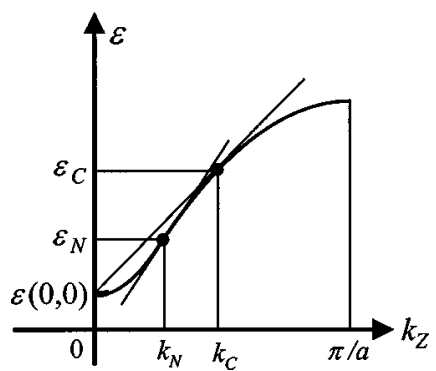

FIG. 5. Characteristic points in a dispersion relation $\varepsilon\left(k_{Z}, 0\right)$ : inflection point $\left(\varepsilon_{N}, k_{N}\right)$ and tangency point $\left(\varepsilon_{C}, k_{C}\right)$. The NEM section starts in the inflection point. Ballistic diode oscillatory regimes start in the tangency point.

branch (based on the single lowest SL2 miniband) in a sufficiently wide energy interval. Therefore we need a sufficiently large SL2 confinement (that is, we need large values of $\varepsilon_{b}$ ). Second, since the calculated dispersion relation $\varepsilon$ $=\varepsilon\left(k_{Z}\right)$ is periodic with half period $\pi / a$, we would like to have the sufficiently extended NEM section $\left(k_{N}, \pi / a\right)$ and also the extended section $\left(k_{C}, \pi / a\right)$ started by a tangency point at $k=k_{C}$ (see Fig. 5). [The above-mentioned tangency point is a starting point of the oscillatory regime in ballistic NEM diodes. ${ }^{4,5}$ To reach a well-developed oscillatory regime, we need a noticeable section $\left(k_{C}, \pi / a\right)$.] To obtain small values of $k_{N}$ and especially $k_{C}$, it is necessary to combine a small effective mass $m_{1}$ in L1 with a sufficiently small height of the effective barrier $\delta_{\text {eff }}=\varepsilon_{\mathrm{SL}}(0,0)$ (see Figs. 3 and 4). An effective width of the miniband, $2 \Delta$, must not be too small, otherwise power-generation potential of the structure will be decreased. As a rule, two-material structures (like GaAs/AlGaAs or InGaAs/InAlAs) cannot satisfy such a combination of requirements. In such two-material structures, QW L1 and SL2 QWs $c$ (Fig. 2) are grown from the same material, and we have no possibility to decrease $\delta_{\text {eff }}$ $=\varepsilon_{\mathrm{SL}}(0,0)$ at the expense of the energy discontinuity $\delta^{\prime}$ (see Figs. 3 and 4). Therefore three-material structures like $\mathrm{Al}_{x} \mathrm{Ga}_{1-x} \mathrm{As} / / \mathrm{GaAs} / \mathrm{Al}_{y} \mathrm{Ga}_{1-y} \mathrm{As}(y>x)$ are optimal. In this structure, $\quad \mathrm{Al}_{x} \mathrm{Ga}_{1-x} \mathrm{As} \quad \mathrm{L} 1$ is combined with GaAs/ $\mathrm{Al}_{y} \mathrm{Ga}_{1-y}$ As SL2. Selecting the value of $y$, we take into account that the top of the SL2 miniband $\left(\delta_{\text {eff }}+2 \Delta\right)$ must be lower than the bottoms of all the $X$ valleys in SL2: $\delta_{\text {eff }}+2 \Delta<\varepsilon_{X}$ (because otherwise electrons transfer to these $X$ valleys across the miniband). The three-material structure considered below with $x=0.15$ and $y=0.5$ satisfies this condition. Turning from $x=0$ (a two-material structure) to $x$ $=0.15$ (a three-material structure) improves the electron dispersion relation $\varepsilon=\varepsilon\left(k_{Z}\right)$ considerably.

Note that currently the CEO technology operates mainly with the GaAs/AlGaAs system where the electron SL2 confinement is not large because of the necessary condition $\delta_{\text {eff }}+2 \Delta<\varepsilon_{X}$. Other material systems require a lot of additional technological efforts in comparison with the simplest GaAs/AlGaAs system. Despite this obstacle, we consider dispersion relations for heterostructures grown on InP substrates. The standard isomorphic two-material $\mathrm{In}_{0.53} \mathrm{Ga}_{0.47} \mathrm{As} / \mathrm{In}_{0.52} \mathrm{Al}_{0.48} \mathrm{As}$ structures with $\varepsilon_{b} \cong 0.51 \mathrm{eV}$ do 
not promise substantial advantages in comparison with the two-material GaAs/AlGaAs system. As a result, one more isomorphic two-material system, ${ }^{17-19}$ $\mathrm{In}_{0.53} \mathrm{Ga}_{0.47} \mathrm{As} / \mathrm{AlAs}_{0.56} \mathrm{Sb}_{0.44}$, grown on InP substrates and with the conduction band offset $\varepsilon_{b} \cong 1.74 \mathrm{eV}$, is also not very promising. (To evaluate a realistic electron dispersion relation in such a superlattice, it is necessary to take into account electron tunneling across valence bands because of the very large $\varepsilon_{b}$. )

The most promising results can be obtained for the three-material strained structure based on a strain-balanced $\mathrm{In}_{x} \mathrm{Ga}_{1-x} \mathrm{As} / \mathrm{In}_{y} \mathrm{Al}_{1-y} \mathrm{As}$ superlattice, grown on an InP substrate with $x>0.53$ and $y<0.52$. The values of $x$ and $y$ as well as widths of SL2 layers $a_{b}$ and $a_{c}$ are selected so that a planar 2D compression of the $\operatorname{In}_{x} \mathrm{Ga}_{1-x} \mathrm{As}-\mathrm{QW}$ compensates a planar $2 \mathrm{D}$ tension of the $\operatorname{In}_{y} \mathrm{Al}_{1-y} \mathrm{As}$ barrier. The third material in this system is a material of L1 overgrown on the cleaved edge of the above-described strain-balanced SL2. This material for approximately equal thicknesses, $a_{b}$ and $a_{c}$, of the SL2 layers can be close to $\operatorname{In}_{0.53} \mathrm{Ga}_{0.47} \mathrm{As}$. In any case, $\operatorname{In}_{z} \mathrm{Ga}_{1-z}$ As with the calculated value $z<x$ can be selected as this third material. The above-mentioned strainbalanced superlattices (of course, without the overgrown edge L1) have been grown repeatedly and successfully. Specifically, they have been used in order to increase the electron confinement in cascade lasers ${ }^{20,21}$ and in infrared multi-QW photodetectors. ${ }^{22}$ The values of $\varepsilon_{b}$ in these strainbalanced structures substantially exceed the abovementioned value $0.51 \mathrm{eV}: \varepsilon_{b}=0.78 \mathrm{eV}$ is mentioned in Refs. 20 and 21 , and $\varepsilon_{b} \cong 0.9 \mathrm{eV}$ is reached in Ref. 22. In accordance to Ref. 16 , the values of $\varepsilon_{b}$ in this system can be even greater. In our calculations here, we use $\varepsilon_{b}=1.00 \mathrm{eV}$.

The strain-balanced $\operatorname{In}_{x} \mathrm{Ga}_{1-x} \mathrm{As} / \mathrm{In}_{y} \mathrm{Al}_{1-y} \mathrm{As}$ superlattices with $x>0.53$ and $y<0.52$ are not the only form of the strain-balanced SLs. The most extended area of such structures is a $\mathrm{Si} / \mathrm{Si}_{1-z} \mathrm{Ge}_{z}$ system, in which only strained structures are possible ${ }^{23}$ and they can be grown on the so-called virtual $\mathrm{Si}_{1-z^{\prime}} \mathrm{Ge}_{z^{\prime}}$ substrates ${ }^{24}$ with $z^{\prime}<z$. Unfortunately, in this system the hole QRST is the most interesting phenomenon, but hole systems are beyond our interest in this article.

As a result of the cleavage of the strain-balanced SL, a cleaved edge surface is nonflat. ${ }^{25,26}$ This nonflatness must be noticeably smoothed out as a result of the assumed secondary overgrowth on this edge surface. The nonflatness effect must be especially small for small spatial periods of the SL assumed here. Note that a certain successful experience of overgrowth on the cleaved edges of strained $\mathrm{In}_{x} \mathrm{Ga}_{1-x} \mathrm{As} / \mathrm{GaAs}-$ and $\mathrm{In}_{x} \mathrm{Ga}_{1-x} \mathrm{As} / \mathrm{AlGaAs}-\mathrm{multi}-\mathrm{QW}$ structures is described in the works of Sakaki with coauthors. $^{27-29}$ In our calculations, we do not take into account the above-mentioned nonflatness of the strained heterobarrier L1/SL2 and consider it analogous to isomorphic structures.

\section{B. Results for the 1D model}

Here we use the 1D model to calculate an electron dispersion relation $\varepsilon\left(k_{Z}, k_{Y}\right)$ for the three-layer heterostructure depicted in Fig. 3. In this heterostructure, we take into ac- count electric fields $E_{1}$ and $E_{2}$ in L1 and SL2, respectively, but assume that $E_{3}=0$ in the barrier layer 3. The problem with the boundary conditions in the form of Eqs. (6a) and (6b) at $X=0$ and the analogous conditions at $X=w_{1}$ is reduced to the transcendent equation:

$$
\begin{aligned}
A i\left(p_{3}\right)\left\{\left[A i^{\prime}\left(p_{1}\right) B i^{\prime}\left(p_{2}\right)-A i^{\prime}\left(p_{2}\right) B i^{\prime}\left(p_{1}\right)\right]\right. \\
\left.\quad+S_{1}\left[A i^{\prime}\left(p_{2}\right) B i\left(p_{1}\right)-A i\left(p_{1}\right) B i^{\prime}\left(p_{2}\right)\right]\right\}+S_{2} A i^{\prime}\left(p_{3}\right) \\
\quad \times\left\{\left[A i\left(p_{2}\right) B i^{\prime}\left(p_{1}\right)-A i^{\prime}\left(p_{1}\right) B i\left(p_{2}\right)\right]\right. \\
\left.\quad+S_{1}\left[A i\left(p_{1}\right) B i\left(p_{2}\right)-A i\left(p_{2}\right) B i\left(p_{1}\right)\right]\right\} \\
\quad=0 .
\end{aligned}
$$

Here:

$$
\begin{aligned}
S_{1}= & \left(m_{1} / m_{3}\right) \lambda_{3} /\left(e \mu_{1} E_{1}\right)^{1 / 3}, \\
S_{2}= & \left(m_{1} / m_{\perp}\right)^{2 / 3}\left(\kappa_{D 1} / \kappa_{D 2}\right)^{1 / 3}, \\
p_{1}= & \left(k_{T}^{2}-\mu_{1} \varepsilon\right) /\left(e \mu_{1} E_{1}\right)^{2 / 3}, \\
p_{2}= & w_{1}\left(e \mu_{1} E_{1}\right)^{1 / 3}-\left(k_{T}^{2}-\mu_{1} \varepsilon\right) /\left(e \mu_{1} E_{1}\right)^{2 / 3}, \\
k_{T}^{2}= & k_{Y}^{2}+k_{Z}^{2}, \\
p_{3}= & \frac{k_{Y}^{2}-\mu_{\perp}\left[\varepsilon-\varepsilon_{\mathrm{SL}}\left(k_{Z}, 0\right)\right]}{\left[\left(e \mu_{1} E_{1}\right)^{2 / 3}\left(\kappa_{D 1} / \kappa_{D 2}\right)^{2 / 3}\left(m_{\perp} / m_{1}\right)^{2 / 3}\right]} \\
& +w_{1}\left(m_{\perp} / m_{1}\right)^{1 / 3}\left(\kappa_{D 2} / \kappa_{D 1}\right)^{2 / 3}\left(e \mu_{1} E_{1}\right)^{1 / 3}, \\
\lambda_{3}^{2}= & \mu_{3}\left(\varepsilon_{3}-\varepsilon\right)+k_{T}^{2}, \text { and } \mu_{1,3, \perp}=2 m_{1,3, \perp} / \hbar^{2}, m_{3}
\end{aligned}
$$

is an isotropic effective mass in the barrier layer 3 (L3), $\varepsilon_{3}$ is a height of the heterobarrier in the plane $X=w_{3}, \kappa_{D 1}$ is a dielectric constant for L1, $\kappa_{D 2}$ is a transverse dielectric constant for SL2, determined by the formula $\kappa_{D 2}=\left(\kappa_{D b} a_{b}\right.$ $\left.+\kappa_{D c} a_{c}\right) /\left(a_{b}+a_{c}\right)$, and $\kappa_{D 2} E_{2}=\kappa_{D 1} E_{1}$. Functions $A i(p)$ and $B i(p)$ in Eq. (12) are Airy functions, determined in Ref. 30, $A i^{\prime}(p)=d A i(p) / d p$ and $B i^{\prime}(p)=d B i(p) / d p$.

The equation, which is analogous to Eq. (12) and determines $\varepsilon\left(k_{Z}, k_{Y}\right)$ for the four-layer subsidiary heterostructure depicted in Fig. 4 with the same parameters of both outer layers $\left(m_{3}=m_{4}, \kappa_{D 3}=\kappa_{D 4}\right)$, is

$$
\begin{aligned}
\left(\frac{\lambda_{3}}{m_{3}}\right. & \left.+\frac{\kappa_{1}}{m_{1}} \cot \kappa_{1} w_{1}\right)\left(\frac{\lambda_{3}}{m_{3}}+\frac{\lambda_{2}}{m_{\perp}} \operatorname{coth} \lambda_{2} w_{2}\right) \\
& \times\left(\frac{\kappa_{1}}{m_{1}} \cot \kappa_{1} w_{1}+\frac{\lambda_{2}}{m_{\perp}} \operatorname{coth} \lambda_{2} w_{2}\right) \\
& -\left(\frac{\lambda_{2}}{m_{\perp}}\right)^{2} \frac{1}{\sinh ^{2} \lambda_{2} w_{2}}\left(\frac{\lambda_{3}}{m_{3}}+\frac{\kappa_{1}}{m_{1}} \cot \kappa_{1} w_{1}\right) \\
& -\left(\frac{\kappa}{m_{1}}\right)^{2} \frac{1}{\sin ^{2} \kappa w_{1}}\left(\frac{\lambda_{3}}{m_{3}}+\frac{\lambda_{2}}{m_{\perp}} \operatorname{coth} \lambda_{2} w_{2}\right) \\
& =0 .
\end{aligned}
$$

Here: $\quad \lambda_{3}^{2}=\mu_{3}\left(\varepsilon_{3}-\varepsilon\right)+k_{T}^{2}, \quad \lambda_{2}^{2}=\mu_{\perp}\left[\varepsilon_{\mathrm{SL}}\left(k_{Z}, 0\right)-\varepsilon\right]+k_{Y}^{2}$, and $\kappa^{2}=\mu_{1} \varepsilon-k_{T}^{2}$.

In Fig. 6, the dispersion relations $\varepsilon\left(k_{Z}, 0\right)$ calculated on the basis of Eq. (12) are presented at several values of the electric field $E_{1}$. They are calculated for the three-material $\mathrm{Al}_{0.15} \mathrm{Ga}_{0.85} \mathrm{As} / / \mathrm{GaAs} / \mathrm{Al}_{0.5} \mathrm{Ga}_{0.5} \mathrm{As}$ structure where 


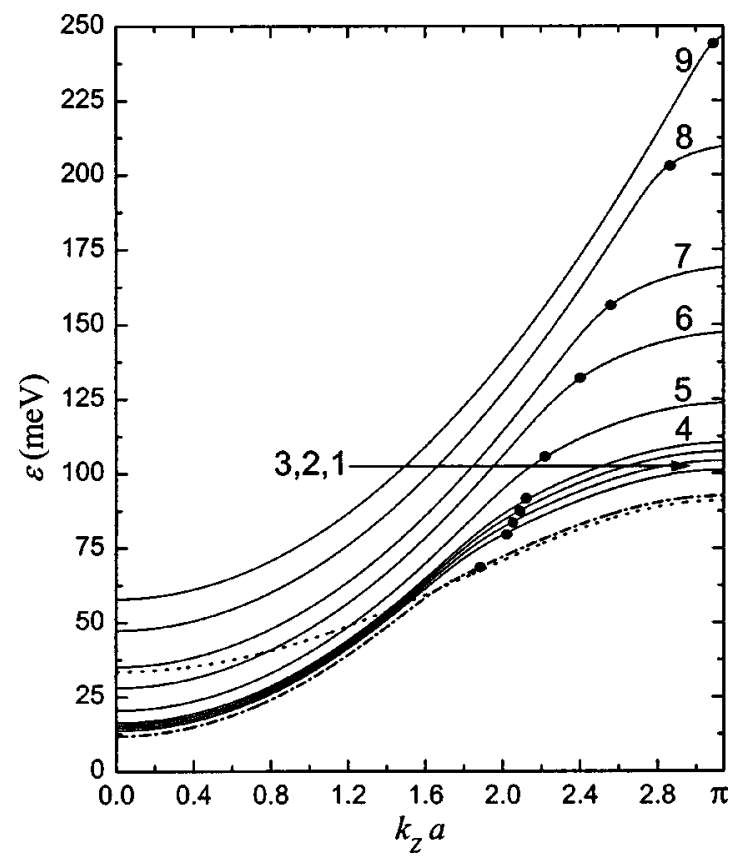

FIG. 6. Electron dispersion relations $\varepsilon\left(k_{Z}, 0\right)$ for the isomorphic threematerial $\mathrm{Al}_{0.15} \mathrm{Ga}_{0.85} \mathrm{As} / / \mathrm{GaAs} / \mathrm{Al}_{0.5} \mathrm{Ga}_{0.5}$ As structures (all the material and structural parameters of the calculated structures are indicated in Table I). The solid curves relate to the 2DEG containing structure (with the potential profile depicted in Fig. 3) for $w_{1}=15 \mathrm{~nm}$ and $E_{1}=2$ (1), 3 (2), 4 (3), 5 (4), 10 (5), 20 (6), 30 (7), 50 (8), and $70 \mathrm{kV} / \mathrm{cm}$ (9). The dash-dotted curve relates to the subsidiary structure with $w_{2}=3 w_{1}$. Dots in the dispersion curves indicate positions of the tangency points $\left(\varepsilon_{C}, k_{C}\right)$. The dotted curve is the dispersion relation $\varepsilon_{\mathrm{SL}}\left(k_{\mathrm{Z}}, 0\right)$ for the GaAs/ $\mathrm{Al}_{0.5} \mathrm{Ga}_{0.5} \mathrm{As}$ SL used in the considered structure. All the results are calculated for the $1 \mathrm{D}$ model.

$\mathrm{Al}_{0.15} \mathrm{Ga}_{0.85} \mathrm{As} \mathrm{L} 1$ with $w_{1}=15 \mathrm{~nm}$ is overgrown on the edge of GaAs $/ \mathrm{Al}_{0.5} \mathrm{Ga}_{0.5} \mathrm{As}$ SL2 with $a_{c}=a_{b}=2.5 \mathrm{~nm}$. All the parameters of this structure used for the calculations are presented in Table I. For comparison, in Fig. 6, the dispersion relation $\varepsilon\left(k_{Z}, 0\right)$ calculated on the basis of Eq. (13) is presented for the subsidiary four-layer structure with the same material and structure parameters and with $w_{2}=3 w_{1}$. The SL2 miniband is also depicted. As it is seen, with a decrease in field $E_{1}$, the electron dispersion relation for 2DEG nears a certain limiting form, which is close to but somewhat differs from the subsidiary structure dispersion relation. There exists a certain limiting value of the tangency point wave vector $k_{C}$, which is achievable at $E_{1} \rightarrow 0$, but we have a very weak field control at low field values. At high field values, the field presses the 2DEG to the barrier plane $X=w_{3}$, and we have

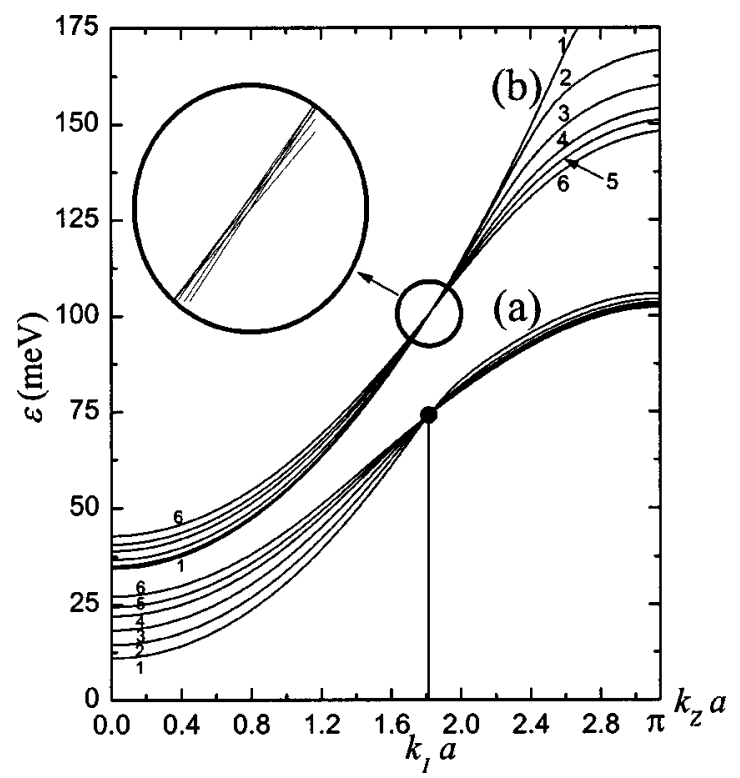

FIG. 7. Electron dispersion relations $\varepsilon\left(k_{Z}, 0\right)$ for the same $2 \mathrm{DEG}$ containing isomorphic structures as considered in Fig. 6 for $E_{1}=3 \mathrm{kV} / \mathrm{cm}$ (a), 30 $\mathrm{kV} / \mathrm{cm}(\mathrm{b})$, and $w_{1}=20$ (1), 15 (2), 12 (3), 10 (4), 9 (5), and $8 \mathrm{~nm}$ (6). The neighborhood of the pseudounited point for $E_{1}=30 \mathrm{kV} / \mathrm{cm}$ is depicted on the enlarged scale in the inset. All the results are calculated for the $1 \mathrm{D}$ model.

the usual subband of the ordinary 2DEG quantization. A real field control of the dispersion relation takes place in very comfortable electric fields $E_{1}=3-10 \mathrm{kV} / \mathrm{cm}$. Remember that this dispersion relation control means an oscillatory regime control in ballistic diode NEM oscillators.

The same as in Fig. 6, dispersion relations are presented in Fig. 7 at the two values of the field, $E_{1}=3$ and $30 \mathrm{kV} / \mathrm{cm}$, and for different thicknesses, $w_{1}$, of L1. It is seen that an increase in $w_{1}$ accentuates the NEM section in the neighborhood of the point $k_{Z}=k_{C}$ not indicated in Fig. 7. The most characteristic specificity of the dispersion relation family depicted in Fig. 7(a) is the "united" point of intersection at $k_{Z}=k_{1}$. This "united" point takes place in the most evident form at $E_{1}=3 \mathrm{kV} / \mathrm{cm}$ as a result of the strong inequality

$$
e E_{1} w_{1} \ll \varepsilon\left(k_{1}\right) \text {. }
$$

For $E_{1}=30 \mathrm{kV} / \mathrm{cm}$, such a "point" is noticeably dispersed. As is seen clearly in the inset in Fig. 7, it consists of a number of neighboring but completely separated intersection points.

TABLE I. Parameters of the three-material structure $\mathrm{Al}_{0.15} \mathrm{Ga}_{0.85} \mathrm{As} / / \mathrm{GaAs} / \mathrm{Al}_{0.5} \mathrm{Ga}_{0.5} \mathrm{As}$.

\begin{tabular}{|c|c|c|c|c|c|c|c|}
\hline \multicolumn{2}{|c|}{ L1: $\mathrm{Al}_{0.15} \mathrm{Ga}_{0.85} \mathrm{As}$} & \multicolumn{4}{|c|}{ SL2: $\mathrm{GaAs} / \mathrm{Al}_{0.5} \mathrm{Ga}_{0.5} \mathrm{As} a_{b}=25 \AA a_{c}=25 \AA$} & \multicolumn{2}{|c|}{ L3: $\mathrm{Al}_{0.5} \mathrm{Ga}_{0.5} \mathrm{As}$} \\
\hline$m_{1} / m_{0}$ & $\kappa_{D 1}$ & $m_{c} / m_{0}$ & $\kappa_{D c}$ & $m_{b} / m_{0}$ & $\kappa_{D b}$ & $m_{3} / m_{0}$ & $\kappa_{D 3}$ \\
\hline 0.076 & 12.43 & 0.063 & 12.85 & 0.107 & 11.46 & 0.107 & 11.46 \\
\hline$m_{\perp} / m_{0}$ & $\kappa_{D 2}$ & \multicolumn{3}{|c|}{$\delta_{\mathrm{eff}}=\varepsilon_{\mathrm{SL}}(0,0)(\mathrm{eV})$} & $\varepsilon_{b}(\mathrm{eV})$ & $\delta^{\prime}(\mathrm{eV})$ & $\varepsilon_{3}(\mathrm{eV})$ \\
\hline 0.045 & 12.16 & \multicolumn{3}{|c|}{0.033} & 0.494 & 0.15 & 0.344 \\
\hline
\end{tabular}

Note: Parameters of the three-material structure $\mathrm{Al}_{0.15} \mathrm{Ga}_{0.85} \mathrm{As} / / \mathrm{GaAs} / \mathrm{Al}_{0.5} \mathrm{Ga}_{0.5} \mathrm{As} m_{1}, m_{c}, m_{b}, m_{3}, \kappa_{D 1}$, $\kappa_{D c}, \kappa_{D b}$, and $\kappa_{D 3}$ were taken from Ref. 31 and $\varepsilon_{b}, \delta^{\prime}$, and $\varepsilon_{3}$ from Ref. 32. Parameters $m_{\perp}, \kappa_{D 2}$, and $\delta_{\text {eff }}$ are the results of our calculations. 


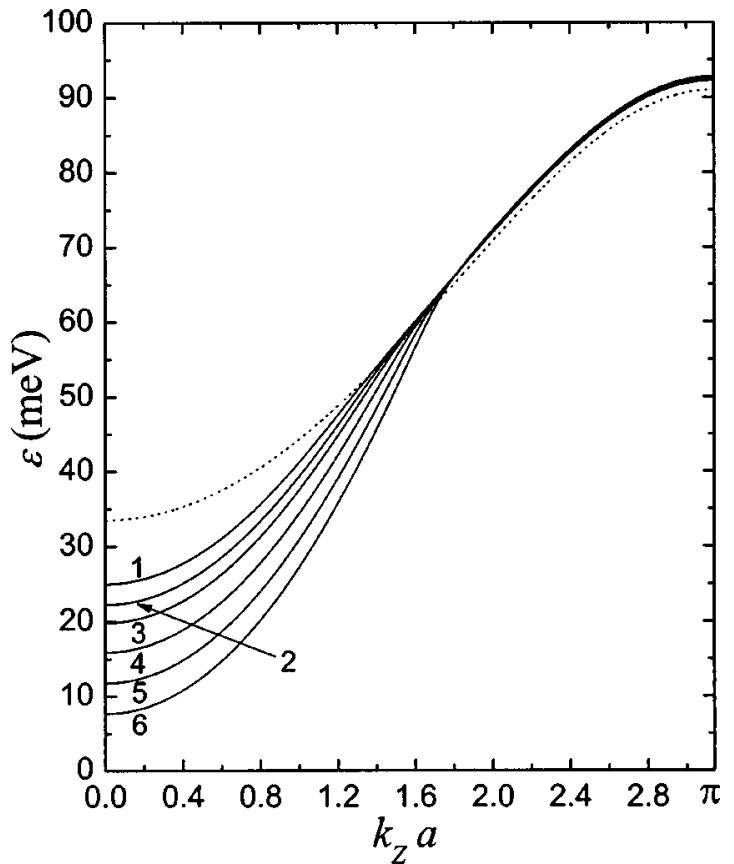

FIG. 8. Electron dispersion relations $\varepsilon\left(k_{Z}, 0\right)$ for the same subsidiary structures as considered in Fig. 6 at $w_{1}+w_{2}=70 \mathrm{~nm}$ and $w_{1}=20$ (6), 15 (5), 12 (4), 10 (3), 9 (2), and $8 \mathrm{~nm}$ (1). All the results are calculated for the 1D model.

Dispersion relation curves $\varepsilon\left(k_{Z}, 0\right)$ for the subsidiary three-material $\mathrm{Al}_{0.15} \mathrm{Ga}_{0.85} \mathrm{As} / / \mathrm{GaAs} / \mathrm{Al}_{0.5} \mathrm{Ga}_{0.5} \mathrm{As}$ structure (Fig. 4) with the same material parameters indicated in Table I are shown in Fig. 8. They are calculated for several values of $w_{1}$ and for $w_{1}+w_{2}=70 \mathrm{~nm}$. Though Fig. 8 qualitatively reminds us of Fig. 7, there are noticeable distinctions. The dispersion relations in Fig. 8 have no so-called united points (which would be similar to that shown in Fig. 7 at $k_{Z}=k_{1}$ ) but they have the united envelope curve, to which they tend asymptotically at large $k_{Z}$. The lowest SL2 miniband serves as this envelope curve.

The same dispersion relation curves as in Figs. 6 and 7 but for another three-material structure, precisely for the strained $\mathrm{In}_{0.53} \mathrm{Ga}_{0.47} \mathrm{As} / / \mathrm{In}_{x} \mathrm{Ga}_{1-x} \mathrm{As} / \mathrm{In}_{y} \mathrm{Al}_{1-y} \mathrm{As}$ structure with $\mathrm{In}_{0.53} \mathrm{Ga}_{0.47} \mathrm{As} \mathrm{L} 1$ and with strain-balanced $\mathrm{In}_{x} \mathrm{Ga}_{1-x} \mathrm{As}_{\mathrm{S}} / \mathrm{In}_{y} \mathrm{Al}_{1-y} \mathrm{As}$ SL2 $(x>0.53, y<0.52)$, are presented in Figs. 9 and 10. All the material and structure parameters used to calculate dispersion relation curves shown in Figs. 9 and 10 are collected in Table II. Actually, we have completed calculations for the two versions of such strainbalanced SLs: (a) $\operatorname{In}_{0.74} \mathrm{Ga}_{0.26} \mathrm{As} / \mathrm{In}_{0.08} \mathrm{Al}_{0.92} \mathrm{As}$ SL2 and (b) $\mathrm{In}_{0.665} \mathrm{Ga}_{0.335} \mathrm{As} / \mathrm{In}_{0.28} \mathrm{Al}_{0.72} \mathrm{As} \quad \mathrm{SL} 2$ with $a=5 \mathrm{~nm}, a_{b}$ $=1.8 \mathrm{~nm}$, and $a_{c}=3.2 \mathrm{~nm}$. The completed calculations are illustrative in their nature and therefore very approximate. More corrected calculations should not be restricted by the framework of the simple KP model and should be based on the multiband approach. We have taken into account variations of effective masses in the SL2 barriers and QWs but we intentionally have not changed the single selected value of $\varepsilon_{b}=1.00 \mathrm{eV}$. Since this value is twice as large as the value used for $\mathrm{GaAs} / \mathrm{Al}_{0.5} \mathrm{Ga}_{0.5} \mathrm{As}$ SL2, we obtain certain advantages. The area of electric field $E_{1}$ control over the electron
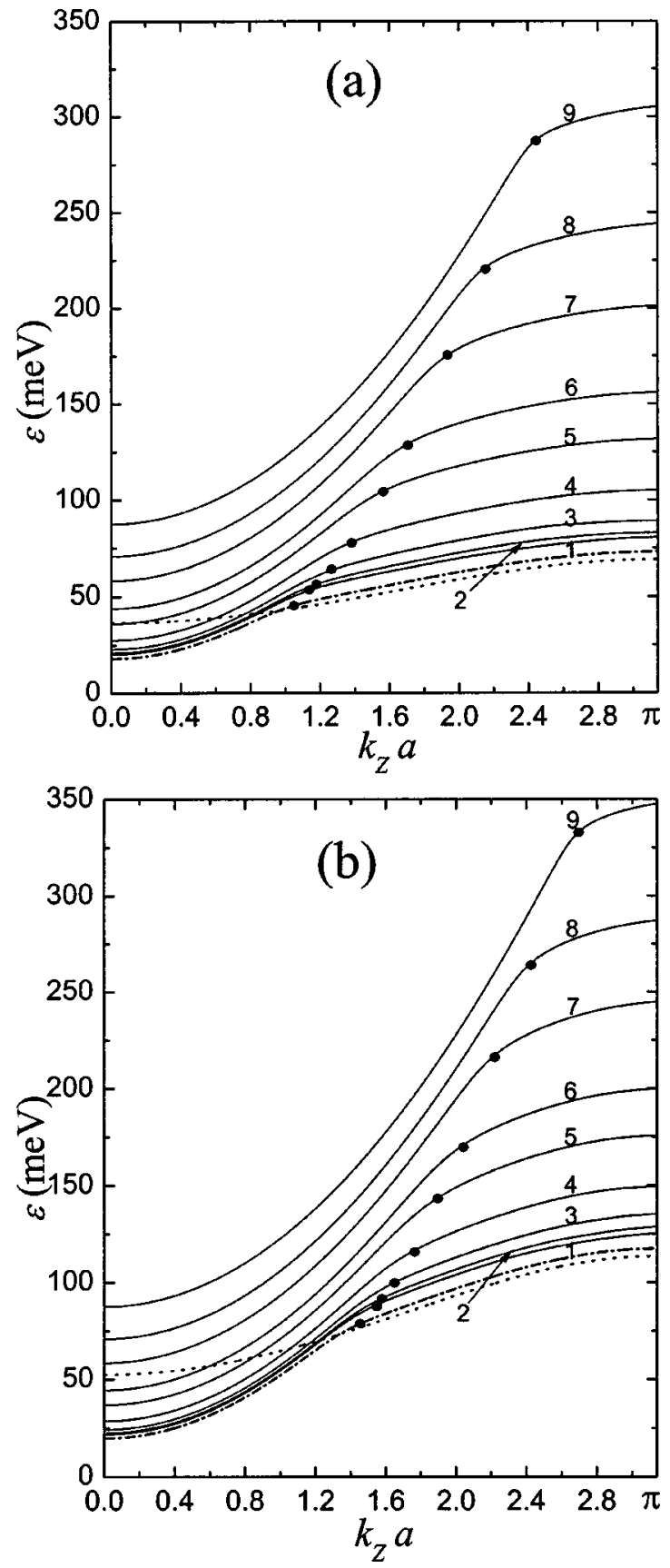

FIG. 9. Electron dispersion relations $\varepsilon\left(k_{Z}, 0\right)$ for 2DEG in the strained three-material $\operatorname{In}_{0.53} \mathrm{Ga}_{0.47} \mathrm{As} / / \mathrm{In}_{x} \mathrm{Ga}_{1-x} \mathrm{As}_{\mathrm{S}} / \mathrm{In}_{y} \mathrm{Al}_{1-y} \mathrm{As}$ structures with $x$ $=0.74, y=0.08$ (a) and $x=0.665, y=0.24$ (b) (all the material and structural parameters of the calculated structures are indicated in Table II). The solid curves relate to the 2DEG containing structure (with the potential profile depicted in Fig. 3) for $w_{1}=15 \mathrm{~nm}$ and $E_{1}=2.2$ (1), 3 (2), 5 (3), 10 (4), 20 (5), 30 (6), 50 (7), 70 (8), and $100 \mathrm{kV} / \mathrm{cm}$ (9). The dash-dotted curve relates to the subsidiary structure with $w_{2}=3 w_{1}$. Dots in the dispersion curves indicate positions of the tangency points $\left(\varepsilon_{C}, k_{C}\right)$. The dotted curve is the dispersion relation $\varepsilon_{\mathrm{SL}}\left(k_{Z}, 0\right)$ for the strain-balanced $\mathrm{In}_{x} \mathrm{Ga}_{1-x} \mathrm{As} / \mathrm{In}_{y} \mathrm{Al}_{1-y} \mathrm{As}$ SL used in the considered structure. All the results are calculated for the $1 \mathrm{D}$ model.

dispersion relations becomes noticeably wider. The values of characteristic wave numbers $k_{N}, k_{C}$, and $k_{1}$ become noticeably smaller. This means that spreading the CEO technology over new (for this technology) material systems can be fruitful. 


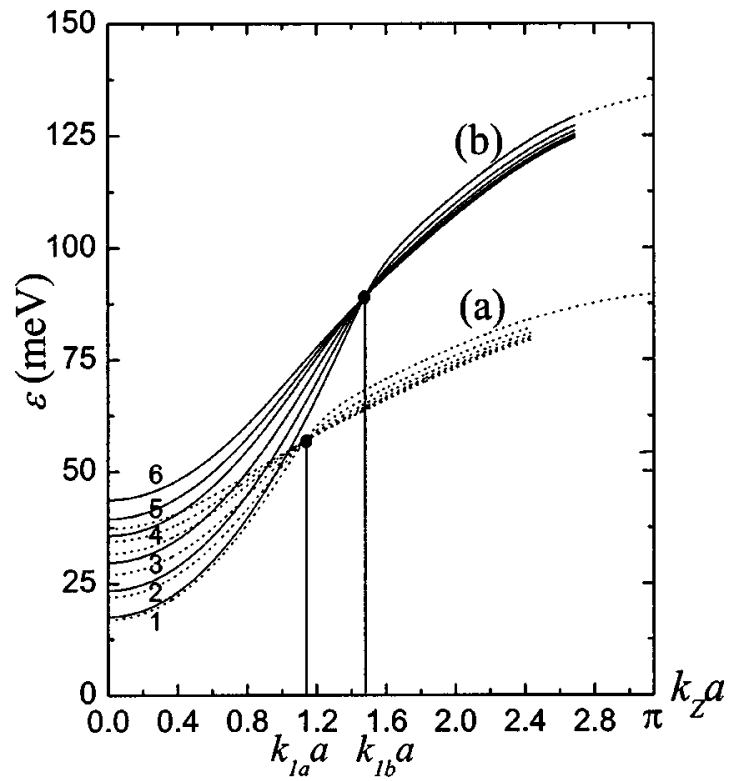

FIG. 10. Electron dispersion relations $\varepsilon\left(k_{Z}, 0\right)$ for the same 2DEG containing strained structures as considered in Fig. 9 for $E_{1}=4 \mathrm{kV} / \mathrm{cm}$ and $w_{1}$ $=20$ (1), 15 (2), 12 (3), 10 (4), 9 (5), and $8 \mathrm{~nm}$ (6). All the results are calculated for the $1 \mathrm{D}$ model.

\section{Comparison of results for 1D and 2D models}

Since 1D model calculations are substantially simpler than 2D model ones, we compared only some of our 1Dmodel results with 2D-model results. In Fig. 11, electron dispersion relations are presented for the unstrained subsidiary two-material $\mathrm{In}_{0.53} \mathrm{Ga}_{0.47} \mathrm{As} / \mathrm{In}_{0.52} \mathrm{Al}_{0.48}$ As structure with $\varepsilon_{b} \cong 0.51 \mathrm{eV}$. All the employed material and structure parameters are given in Table III. The dispersion curves are calculated for both 2D-model and 1D-model versions. As it is seen, the results for both versions are well correlated. There are small quantitative discrepancies for $\varepsilon(0,0)$ and in the neighborhood of $k_{Z}=\pi / a$. Discrepancies can be larger if the conditions $w_{1,2} \gg a, a_{c, b}$ are poorly satisfied.

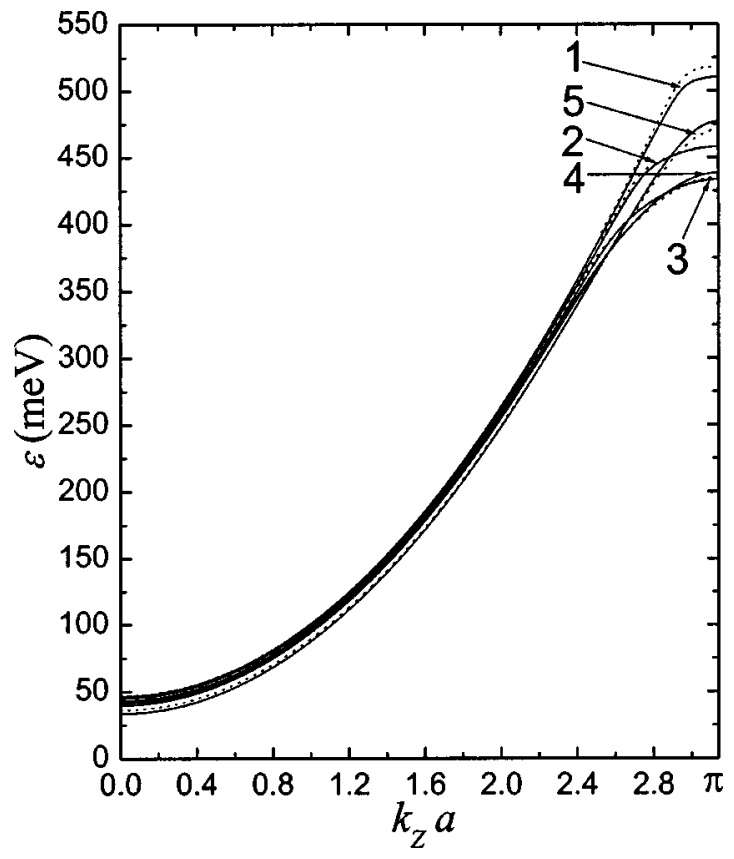

FIG. 11. Electron dispersion relations $\varepsilon\left(k_{Z}, 0\right)$ for the isomorphic subsidiary $\mathrm{In}_{0.53} \mathrm{Ga}_{0.47} \mathrm{As} / / \mathrm{In}_{0.53} \mathrm{Ga}_{0.47} \mathrm{As} / \mathrm{In}_{0.52} \mathrm{Al}_{0.48} \mathrm{As}$ structure (all the material and structural parameters of the calculated structures are indicated in Table III). The solid curves are the 1D-model results and the dotted curves are the 2D-model results for the same parameters. Curves are calculated for $a$ $=a_{c}+a_{b}=4 \mathrm{~nm}, a_{c}=1.5$ (1), 2.0 (2), 2.5 (3), 3.0 (4), and $3.5 \mathrm{~nm}$ (5).

There are several situations when the 1D model is unsuitable in principle. For example, consider a heterostructure where a homogeneous semiconductor layer (L1) is placed between two superlattices (SL1 and SL2; see Fig. 12). Assume that both SLs are identical (with equal $\varepsilon_{b}, a_{b}$, and $\left.a_{c}\right)$. Then the $1 \mathrm{D}$ model leads to the single dispersion relation picture for the determined collection of the parameters, whereas the 2D model leads to different pictures for symmetric [Fig. 12(a)] and asymmetric [Fig. 12(b)] cases as well as for the variety of intermediate cases. Of course, these differ-

TABLE II. Parameters of the three-material structures $\mathrm{In}_{0.53} \mathrm{Ga}_{0.47} \mathrm{As} / / \mathrm{In}_{0.74} \mathrm{Ga}_{0.26} \mathrm{As} / \mathrm{In}_{0.08} \mathrm{Al}_{0.92} \mathrm{As}$ and $\mathrm{In}_{0.53} \mathrm{Ga}_{0.47} \mathrm{As} / / \mathrm{In}_{0.665} \mathrm{Ga}_{0.335} \mathrm{As} / \mathrm{In}_{0.28} \mathrm{Al}_{0.72} \mathrm{As}$.

\begin{tabular}{|c|c|c|c|c|c|c|c|}
\hline \multicolumn{2}{|c|}{ L1: $\mathrm{In}_{0.53} \mathrm{Ga}_{0.47} \mathrm{As}$} & \multicolumn{4}{|c|}{ SL2: $\operatorname{In}_{0.74} \mathrm{Ga}_{0.26} \mathrm{As} / \mathrm{In}_{0.08} \mathrm{Al}_{0.92} \mathrm{As}, a_{b}=18 \AA, a_{c}=32 \AA$} & \multicolumn{2}{|c|}{ L3: $\operatorname{In}_{0.52} \mathrm{Al}_{0.48} \mathrm{As}$} \\
\hline$m_{1} / m_{0}$ & $\kappa_{D 1}$ & $m_{c} / m_{0}$ & $\kappa_{D c}$ & $m_{b} / m_{0}$ & $\kappa_{D b}$ & $m_{3} / m_{0}$ & $\kappa_{D 3}$ \\
\hline 0.043 & 14.07 & 0.035 & 15.15 & 0.14 & 10.47 & 0.072 & 12.1 \\
\hline$m_{\perp} / m_{0}$ & $\kappa_{D 2}$ & \multicolumn{3}{|c|}{$\delta_{\mathrm{eff}}=\varepsilon_{\mathrm{SL}}(0,0)(\mathrm{eV})$} & $\varepsilon_{b}(\mathrm{eV})$ & $\delta^{\prime}(\mathrm{eV})$ & $\varepsilon_{3}(\mathrm{eV})$ \\
\hline 0.04 & 13.47 & \multicolumn{3}{|c|}{0.036} & 1.0 & 0.18 & 0.51 \\
\hline \multicolumn{2}{|c|}{ L1: $\mathrm{In}_{0.53} \mathrm{Ga}_{0.47} \mathrm{As}$} & \multicolumn{4}{|c|}{ SL2: $\operatorname{In}_{0.665} \mathrm{Ga}_{0.335} \mathrm{As} / \mathrm{In}_{0.28} \mathrm{Al}_{0.72} \mathrm{As}, a_{b}=18 \AA, a_{c}=32 \AA$} & \multicolumn{2}{|c|}{ L3: $\operatorname{In}_{0.52} \mathrm{Al}_{0.48} \mathrm{As}$} \\
\hline$m_{1} / m_{0}$ & $\kappa_{D 1}$ & $m_{c} / m_{0}$ & $\kappa_{D c}$ & $m_{b} / m_{0}$ & $\kappa_{D b}$ & $m_{3} / m_{0}$ & $\kappa_{D 3}$ \\
\hline 0.043 & 14.07 & 0.0384 & 14.38 & 0.095 & 11.49 & 0.072 & 12.1 \\
\hline$m_{\perp} / m_{0}$ & $\kappa_{D 2}$ & \multicolumn{3}{|c|}{$\delta_{\mathrm{eff}}=\varepsilon_{\mathrm{SL}}(0,0)(\mathrm{eV})$} & $\varepsilon_{b}(\mathrm{eV})$ & $\delta^{\prime}(\mathrm{eV})$ & $\varepsilon_{3}(\mathrm{eV})$ \\
\hline 0.04 & 13.34 & \multicolumn{3}{|c|}{0.052} & 1.0 & 0.18 & 0.51 \\
\hline
\end{tabular}

Note: Parameters of the three-material structures $\mathrm{In}_{0.53} \mathrm{Ga}_{0.47} \mathrm{As} / / \mathrm{In}_{0.74} \mathrm{Ga}_{0.26} \mathrm{As} / \mathrm{In}_{0.08} \mathrm{Al}_{0.92} \mathrm{As}$ and $\mathrm{In}_{0.53} \mathrm{Ga}_{0.47} \mathrm{As} / / \mathrm{In}_{0.665} \mathrm{Ga}_{0.335} \mathrm{As} / \mathrm{In}_{0.28} \mathrm{Al}_{0.72} \mathrm{As} m_{1}, m_{c}, m_{b}, m_{3}, \kappa_{D 1}, \kappa_{D c}, \kappa_{D b}, \kappa_{D 3}, \varepsilon_{b}, \delta^{\prime}$, and $\varepsilon_{3}$ were taken from Ref. 33 (see also Ref. 16). Parameters $m_{\perp}, \kappa_{D 2}$, and $\delta_{\text {eff }}$ are the results of our calculations. 
TABLE III. Parameters of the two-material structure $\mathrm{In}_{0.53} \mathrm{Ga}_{0.47} \mathrm{As} / \mathrm{In}_{0.52} \mathrm{Al}_{0.48} \mathrm{As}$.

\begin{tabular}{|c|c|c|c|c|c|}
\hline \multirow{2}{*}{$\begin{array}{l}\mathrm{L} 1: \mathrm{In}_{0.53} \mathrm{Ga}_{0.47} \mathrm{As} \\
m_{c} / m_{0} \\
\end{array}$} & \multicolumn{4}{|c|}{ SL2: $\operatorname{In}_{0.53} \mathrm{Ga}_{0.47} \mathrm{As} / \mathrm{In}_{0.52} \mathrm{Al}_{0.48} \mathrm{As}, a_{b}+a_{c}=40 \AA$} & \multirow{2}{*}{$\begin{array}{l}\text { L3: } \mathrm{In}_{0.52} \mathrm{Al}_{0.48} \mathrm{As} \\
m_{b} / m_{0}=m_{3} / m_{0}\end{array}$} \\
\hline & \multicolumn{4}{|c|}{$\varepsilon_{b}=\varepsilon_{3}(\mathrm{eV})$} & \\
\hline \multirow[t]{3}{*}{0.043} & \multicolumn{4}{|c|}{0.51} & 0.072 \\
\hline & $a_{c}=15 \AA$ & $a_{c}=20 \AA$ & $a_{c}=25 \AA$ & $a_{c}=30 \AA$ & $a_{c}=35 \AA$ \\
\hline & $a_{b}=25 \AA$ & $a_{b}=20 \AA$ & $a_{b}=15 \AA$ & $a_{b}=10 \AA$ & $a_{b}=5 \AA$ \\
\hline$\delta_{\mathrm{eff}}=\varepsilon_{\mathrm{SL}}(0,0)(\mathrm{eV})$ & 0.288 & 0.223 & 0.166 & 0.113 & 0.059 \\
\hline$m_{\perp} / m_{0}$ & 0.055 & 0.051 & 0.049 & 0.047 & 0.045 \\
\hline
\end{tabular}

Note: Parameters of the two-material structure $\mathrm{In}_{0.53} \mathrm{Ga}_{0.47} \mathrm{As} / \mathrm{In}_{0.52} \mathrm{Al}_{0.48} \mathrm{As} m_{c}, m_{b}, m_{3}, \varepsilon_{b}$, and $\varepsilon_{3}$ were taken from Ref. 33. Parameters $m_{\perp}$ and $\delta_{\text {eff }}$ are the results of our calculations.

ent results are close to each other if $w_{1,2} \gg a, a_{c, b}$ but they are qualitatively different.

The second example relates to $2 \mathrm{DEG}$ in the cleaved SL with a very high (infinite) heterobarrier covering the cleavage. If effective masses in the barriers and QWs forming the SL are equal, the separation of the variables takes place in the corresponding Schrödinger equation, and the SL is quantized as the unified whole. If $m_{b} \neq m_{c}$, such a separation does not occur, and electron energy $\varepsilon\left(k_{Z}, 0\right)-\varepsilon(0,0)$ must depend on the value of the electric field, $E$, forming 2 DEG. Such dependence cannot be observed in the 1D-model calculation, since we deal with the single transverse mass $m_{\perp}$ in this model. But such a difference is observable in the $2 \mathrm{D}$ model calculations (as seen in Fig. 13). The effect must be pronounced for $e E \approx(2 \Delta / a)\left(m_{b}-m_{a}\right) /\left(m_{b}+m_{a}\right)$, and it is confirmed by Fig. 13.

\section{DISCUSSION}

In the preceding section, we have shown that QRST in a system consisting of an ordinary current-conducting QW channel and an SL, on the cleaved edge of which this QW is overgrown, allows one to engineer various electron dispersion relations with NEM sections. Such an NEM section can induce a negative-differential-drift-velocity section in the electric field dependence of a drift velocity and, as a result, serve as a mechanism of Gunn oscillators (instead of the well-known Ridley-Watkins-Hilsum mechanism). In this case, we need to take into account all the necessary scattering processes both inside of the considered selected dispersion branch and with participation of all the other neighboring branches. But QRST is especially effective for short- $n$-base $n^{+} n n^{+}$diodes with a ballistic electron trans-

(a)

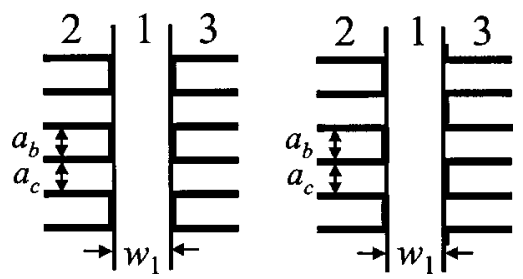

FIG. 12. Hypothetical heterostructures demonstrating insufficiency of the 1D-model approach: (a) the symmetric SL2/L1/SL3 structure, (b) the antisymmetric SL2/L1/SL3 structure. The 1D-model approach does not distinguish between these structures. The 2D-model approach does. port across the $n$ base. To implement such diodes, we need to design short (in comparison to the mean free path length) $n$ bases. The systems containing SL fragments have a doubtless advantage in comparison with all the others because we can bind a number of SL2 periods above by a certain sufficiently small value $N$ during the primary SL1 growth. Of course, to use the macroscopic approach (and specifically the 1D model), we need to keep $N \gg 1$, but if $a=4 \mathrm{~nm}$ and $N=6$ $\gg 1$ we obtain a base length $l=24 \mathrm{~nm}$. This number is smaller than a free path length with emission and absorption of optical phonons at $T=80 \mathrm{~K}$ for the above-considered L1 and SL2 materials. Note that the requirement $N \gg 1$ is only a criterion of the macroscopic approach (but not the QRST mechanism, which can exist for several SL2 periods and even for $N=1$ ).

During the above-mentioned primary SL2 growth, the grown SL2 fragment can be limited on both sides by high resistance regions, which do not allow electrons to spread

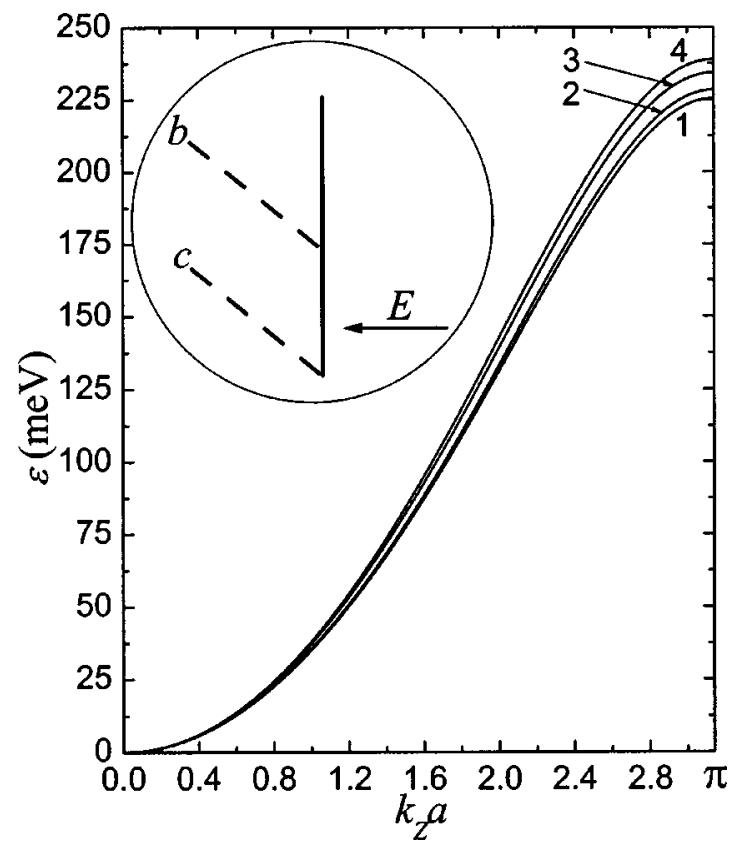

FIG. 13. Electron dispersion relations for the $2 \mathrm{DEG}$ in the half-infinite $\mathrm{In}_{0.53} \mathrm{Ga}_{0.47} \mathrm{As} / \mathrm{In}_{0.52} \mathrm{Al}_{0.48} \mathrm{As} \mathrm{SL}$ medium with the cleaved edge covered by the very high (infinite) potential barrier (see the sketch in the inset). The SL parameters are $\varepsilon_{b}=0.51 \mathrm{eV}, a_{b}=a_{c}=2 \mathrm{~nm}$. The inducing electric field $E$ is equal to 5 (1), 100 (2), 1000 (3), and $2000 \mathrm{kV} / \mathrm{cm}$ (4). These results are calculated for the 2D model. The 1D model does not account for such a field effect. 


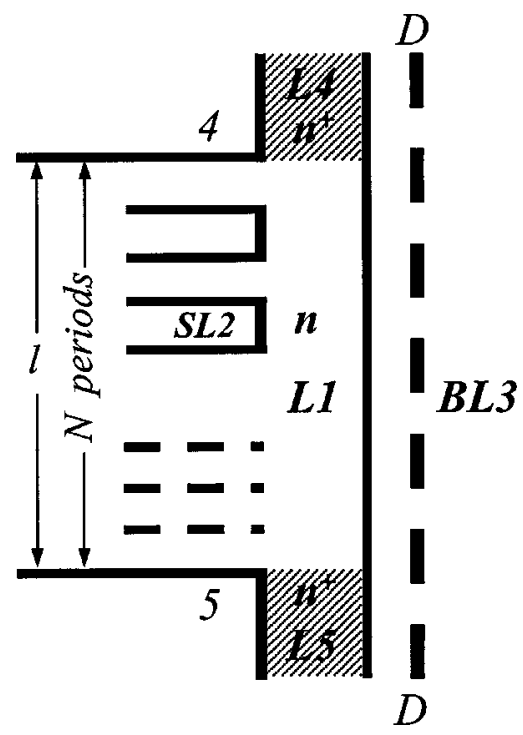

FIG. 14. Short ( $N$ periodic) fragment of the acceptor-doped SL2 grown between two undoped high-ohmic barrier regions 4 and 5. After a cleavage, the homogeneous L1 is overgrown on the cleaved edge and covered by the homogeneous barrier L3 (BL3) with the ionized donor sheet D. Since L1 extensions, L4 and L5, covering the high-ohmic barrier regions 4 and 5 contain all the 2DEG induced by the donor sheet, we have the $n^{+} n n^{+}$-diode structure in the L4/L1/L5 section.

deep inside. One such version has been presented elsewhere. ${ }^{16}$ Now, we consider another variant, which is demonstrated in Fig. 14. Here, the acceptor-doped SL2 fragment with length $l$ is placed between two undoped barrier regions 4 and 5. As above, we assume that the donor modulation in the barrier L3 and the acceptor doping in SL2, $N_{D}$, are selected so that the initial electron concentration in 2DEG would be small (for example, $\sim 0.01 N_{D}$ ). But in L1 sections, which are contacting the undoped regions 4 and 5 (they are indicated by L4 and L5 in Fig. 14), the electron concentration is approximately equal to $N_{D}$. So we obtain the real $n^{+} n n^{+}$-diode structure, and this structure takes place only in the 2DEG layer and does not spread deeper.

\section{CONCLUDING REMARKS}

In this article, we have briefly stated the results of dispersion relation calculations for a structure, which include the QW layer L1 overgrown on the cleaved edge of the acceptor doped SL2 and covered by the barrier L3 (Fig. 2). The latter contains the modulation donor-doping sheet and induces a 2DEG in L1 that spreads into SL2 at large values of wave number $k_{Z}$ (along the SL2 vector). This spreading leads to the QRST manifestation in the form of an increase in an electron effective mass and the appearance of an NEM section in an electron dispersion relation. Such an effect is most highly manifested in the so-called three-material structures, in which a conduction band bottom for electrons in L1 is higher than such a bottom in QWs of SL2 but is lower than in barriers of the same SL2. Two examples of the abovementioned three-material structures have been considered: the isomorphic $\mathrm{Al}_{0.15} \mathrm{Ga}_{0.85} \mathrm{As} / / \mathrm{GaAs} / \mathrm{Al}_{0.5} \mathrm{Ga}_{0.5} \mathrm{As}$ structure and the strained $\mathrm{In}_{0.53} \mathrm{Ga}_{0.47} \mathrm{As} / / \mathrm{In}_{x} \mathrm{Ga}_{1-x} \mathrm{As} / \mathrm{In}_{y} \mathrm{Al}_{1-y} \mathrm{As}$ structure with $\mathrm{In}_{0.53} \mathrm{Ga}_{0.47} \mathrm{As} \mathrm{L} 1$ and with strain-balanced
$\mathrm{In}_{x} \mathrm{Ga}_{1-x} \mathrm{As}_{\mathrm{In}} \mathrm{In}_{y} \mathrm{Al}_{1-y} \mathrm{As}$ SL2 $(x>0.53, y<0.52)$. The best results have been demonstrated for the latter structures, but the results for the three-material structure in the GaAs/ AlGaAs material system are also of interest.

All the main results are obtained for the simplified 1D model. But for a deeper substantiation of these results, we have made several comparisons of the 1D- and 2D-model results with good agreement between the two. We have also presented some examples when the 1D-model simplification is inappropriate.

The cleaved edge overgrowth technology allows one not only to engineer novel electron dispersion relations but to embed the base fragments with such dispersion relations in very short ballistic diode structures and to organize the necessary $n^{+}$-contact cathode and anode regions with very small capacitive connections.

\section{ACKNOWLEDGMENTS}

The authors thank Dr. S. Luryi, Dr. A. Zaslavsky, Dr. R. Beresford, and Dr. H. Eisele for fruitful discussions on problems relating to this work. The authors also thank Dr. S. Tipton for numerous useful notes. This work was supported by the AFOSR through the MURI program F 49620-00-10328 and NSF Grant No. ECS-0099913.

${ }^{1}$ Z. S. Gribnikov, Fiz. Tekh. Poluprovodn. (S.-Peterburg) 6, 1380 (1972) [Sov. Phys. Semicond. 6, 1204 (1973)].

${ }^{2}$ K. Hess, H. Morkoc, H. Shichijo, and B. G. Streetman, Appl. Phys. Lett. 35, 469 (1979).

${ }^{3}$ Z. S. Gribnikov, K. Hess, and G. A. Kosinovsky, J. Appl. Phys. 77, 1337 (1995).

${ }^{4}$ Z. S. Gribnikov and A. N. Korshak, Semiconductors 28, 812 (1994).

${ }^{5}$ Z. S. Gribnikov, A. N. Korshak, and N. Z. Vagidov, J. Appl. Phys. 80, 5799 (1996).

${ }^{6}$ Z. S. Gribnikov, R. R. Bashirov, and V. V. Mitin, IEEE J. Sel. Top. Quantum Electron. 7, 630 (2001).

${ }^{7}$ Z. S. Gribnikov, N. Z. Vagidov, H. Eisele, V. V. Mitin, and G. I. Haddad, Proceedings of the 2001 International Semiconductor Device Research Symposium, Washington, DC, 2001, p. 559.

${ }^{8}$ Z. S. Gribnikov, R. R. Bashirov, H. Eisele, V. V. Mitin, and G. I. Haddad, Physica E (Amsterdam) 12, 276 (2002).

${ }^{9}$ L. Pfeiffer, K. W. West, H. L. Stormer, J. P. Eisenstein, K. W. Baldwin, D. Gershoni, and J. Spector, Appl. Phys. Lett. 56, 1697 (1990).

${ }^{10}$ L. Pfeiffer, H. L. Störmer, K. W. Baldwin, K. W. West, A. R. Goñi, A. Pinczuk, R. S. Ashoori, M. M. Dignam, and W. Wegscheider, J. Cryst. Growth 127, 849 (1993).

${ }^{11}$ W. Wegscheider, L. N. Pfeiffer, A. Pinczuk, K. W. West, M. M. Dignam, R. Hull, and R. E. Leibenguth, J. Cryst. Growth 150, 285 (1995).

${ }^{12}$ A. Majumdar, L. R. Rokhinson, D. C. Tsui, L. N. Pfeiffer, and K. W. West, Appl. Phys. Lett. 76, 3600 (2000).

${ }^{13}$ R. A. Deutschmann, W. Wegscheider, M. Rother, M. Bichler, and G. Arbstreiter, Appl. Phys. Lett. 79, 1564 (2001).

${ }^{14}$ R. A. Deutschmann, W. Wegscheider, M. Rother, M. Bichler, and G. Arbstreiter, Physica E (Amsterdam) 12, 281 (2002).

${ }^{15}$ S.-L. Chuang, Physics of Optoelectronic Devices (Wiley, New York, 1995).

${ }^{16}$ Z. S. Gribnikov, N. Z. Vagidov, and V. V. Mitin, J. Computat. Electron (to be published).

${ }^{17}$ A. Neogi, H. Yoshida, T. Mosume, N. Georgiev, and O. Wada, IEEE J. Sel. Top. Quantum Electron. 7, 710 (2001).

${ }^{18}$ N. Georgiev and T. Mosume, J. Vac. Sci. Technol. B 19, 1747 (2001).

${ }^{19}$ S. Muto and T. Inata, Semicond. Sci. Technol. 9, 1157 (1994).

${ }^{20}$ J. Faist, F. Capasso, D. L. Sivco, A. L. Hutchinson, S.-N. G. Chu, and A. Y. Cho, Appl. Phys. Lett. 72, 680 (1998).

${ }^{21}$ S. Slivken and M. Razeghi, Proceedings of the 2001 International Semiconductor Device Research Symposium, Washington, DC, 2001, 514.

${ }^{22}$ H. Asai and Y. Kawamura, Appl. Phys. Lett. 56, 746 (1990). 
${ }^{23}$ J. C. Bean, Proc. IEEE 80, 571 (1992).

${ }^{24}$ K. Kawaguchi, Y. Shiraki, N. Usami, J. Zhang, N. J. Woods, G. Breton, and G. Parry, Appl. Phys. Lett. 79, 344 (2001).

${ }^{25}$ C. D. Akyüz, H. T. Johnson, A. Zaslavsky, L. B. Freund, and D. A. Syphers, Phys. Rev. B 60, 16597 (1999).

${ }^{26}$ J. Liu, A. Zaslavsky, C. D. Akyüz, B. R. Perkins, and L. B. Freund, Phys. Rev. B 62, 7731 (2000).

${ }^{27}$ H. Akiyama, T. Someya, M. Yoshita, T. Sasaki, and H. Sakaki, Phys. Rev. B 57, 3765 (1998).

${ }^{28}$ M. Yoshita, H. Akiyama, T. Someya, and H. Sakaki, J. Appl. Phys. 83,
3777 (1998)

${ }^{29}$ H. Akiyama, J. Phys.: Condens. Matter 10, 3095 (1998).

${ }^{30}$ Handbook of Mathematical Functions, edited by M. Abramowitz and I. A. Stegun (National Bureau of Standards, 1964).

${ }^{31}$ S. Adachi, J. Appl. Phys. 58, R1 (1985).

${ }^{32}$ L. R. Wilson, P. T. Keightley, J. W. Cockburn, J. P. Duck, M. S. Skolnick, J. C. Clark, G. Hill, M. Moran, and R. Grey, Appl. Phys. Lett. 75, 2079 (1999).

${ }^{33}$ S. Tiwari and D. L. Frank, Appl. Phys. Lett. 60, 630 (1992). 